\title{
Arquitectura de validación de diseños didácticos para la formación de profesores de matemáticas
}

Architecture of Validation of Didactic Designs for the Training of Math Teachers

Arquitetura de validação de projetos didáticos para a formação de professores de matemáticas

\author{
Olga Lucía León-Corredor* (iD) 0000-0003-4373-8630 \\ Jaime Humberto Romero-Cruz** (iD) 0000-0002-7504-6068 \\ Edwin Alfredo Carranza-Vargas*** (iD) 0000-0003-0777-6146 \\ Francisco Alejandro Sánchez-Acero**** (iD) 0000-0003-0265-8749 \\ William Andrey Suárez-Moya**** (iD) 0000-0002-0496-5200 \\ Claudia Cecilia Castro-Cortés ${ }^{* * * * *}$ (iD) 0000-0002-4224-2580 \\ Diana Gil-Chaves****** (ID) 0000-0003-3872-1105 \\ Martha Alba Bonilla-Estévez ${ }^{* * * * * * *}$ (iD) 0000-0002-9694-0325
}

Doctora en Educación. Universidad Distrital Francisco José de Caldas, Bogotá, Colombia. Correo electrónico: olleon@udistrital.edu.co

** Especialista en Educación Matemática. Universidad Distrital Francisco José de Caldas, Bogotá, Colombia Correo electrónico: jaimeedumat@udistrital.edu.co

*** Magíster en Educación y TIC. Universidad Distrital Francisco José de Caldas, Bogotá, Colombia. Correo electrónico: eacarranzav@udistrital.edu.co

...* Magíster en Enseñanza de las Ciencias Exactas y Naturales. Fundación Universitaria Konrad Lorenz. Bogotá, Colombia. Correo electrónico: franciscoa.sancheza@konradlorenz.edu.co

*...** Licenciado en Educación Básica con énfasis en Matemáticas. Universidad Distrital Francisco José de Caldas, Bogotá, Colombia. Correo electrónico: wasuarezm@acorreo.udistrital.edu.co

****** Magíster en Docencia e Investigación Universitaria. Universidad Distrital Francisco José de Caldas, Bogotá, Colombia. Correo electrónico: cccastroc@udistrital.edu.co

****** Candidata a doctora en Educación. Universidad Distrital Francisco José de Caldas, Bogotá, Colombia Correo electrónico: dgilc@udistrital.edu.co

*m**** Magister en Desarrollo Educativo y Social. Universidad Pedagógica Nacional, Bogotá Colombia. Correo electrónico: marthabonilae@gmail.com 


\begin{abstract}
Resumen
Este artículo presenta los resultados del proyecto "Desarrollo didáctico y tecnológico en escenarios didácticos para la formación de profesores que acogen la diversidad". La investigación incorpora una perspectiva teórica del diseño universal y una posición ético-política de una educación matemática con todos. Se entrega como resultado de investigación una arquitectura para validar las hipótesis didácticas que fundamentan el diseño de un ambiente y tres objetos virtuales de aprendizaje. Los diseños didácticos son validados en un curso para formación de profesores de matemáticas de la educación básica con diseño instruccional para la interacción cara a cara y en línea (virtual). La arquitectura como estructura de validación articula las metodologías provenientes de experimento de enseñanza, investigación en diseño y comunidades de práctica. Los resultados se centran en la validación de hipótesis sobre formación de profesores de matemáticas para acoger la diversidad de poblaciones, diseños didácticos accesibles y metodologías para la validación didáctica en comunidades de práctica.
\end{abstract}

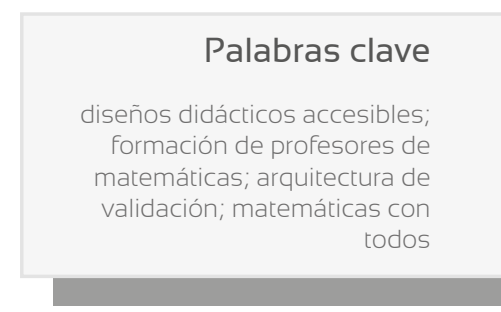

\section{Keywords}

accessible learning designs;

training of mathematics pre-service teachers; validation architecture; mathematic with everyone

\begin{abstract}
This article presents the results of the project "Didactic and technological development in didactic scenarios for the training of teachers who embrace diversity". From a theoretical perspective of the universal design and from a political-ethical position of a mathematical education with everyone, the research provides an architecture as a research result to validate the didactic hypotheses that serve as the basis for the design of an environment and three virtual objects of learning. The didactic designs are validated in a training course for elementary school mathematics teachers, with instructional design for both face-to-face and online interactions. Architecture as a validation structure articulates the methodologies resulting from teaching experiments, design research, and communities of practice. The results focus on the validation of hypotheses about training of pre-service math teachers to accommodate the diversity of populations, accessible didactic designs, and methodologies for didactic validation in communities of practice.
\end{abstract}

\begin{abstract}
Resumo
Este artigo apresenta os resultados do projeto "Desenvolvimento didático e tecnológico em cenários didáticos para a formação de professores que adotam a diversidade". A pesquisa incorpora uma perspectiva teórica do design universal e uma posição ético-política de uma educação matemática com todos. Como resultado de investigação, é apresentada uma arquitetura para validar as hipóteses didáticas que fundamentam o desenho de um ambiente e três objetivos virtuais de aprendizagem. Os projetos didáticos são validados em uma aula para formação de professores de matemáticas de educação básica, com design instrucional para a interação face a face e online. A arquitetura como estrutura de validação articula as metodologias provenientes de experimento de ensino, pesquisa em projeto e comunidades de prática. Os resultados centram-se na validação de hipóteses sobre formação de professores de matemáticas para adotar a diversidade de populações, projetos didáticos acessíveis e metodologias para a validação didática em comunidades de prática.
\end{abstract}

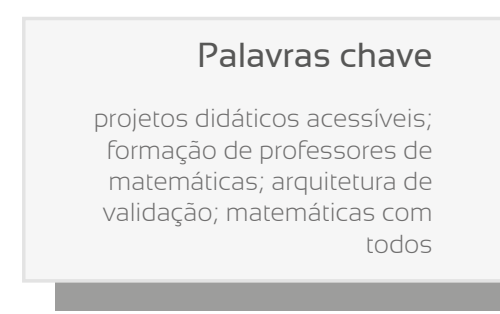

Artículo de investigación
Revista Colombiana de Educación, N.7 73. Segundo semestre de 2017, Bogotá, Colombia.

doi: 10.17227/01203916.73rce233.258

Para citar este artículo: Bonilla, A., Carranza, E., Castro, C., Gil-Chaves, D., León, O., Romero, J., Sánchez, F., y Suárez, W. (2017). Arquitectura de validación de diseños didácticos para la formación de profesores de matemáticas. Revista Colombiana de Educación, (73), 235-260.

N. ${ }^{\circ} 73$ 


\section{Introducción'}

Una de las problemáticas educativas actuales es la marginación de poblaciones de la educación matemática. A pesar de la promulgación de la Declaración Mundial de los Derechos Humanos, hace 67 años, la Declaración Mundial de Educación para Todos, hace 25 años, y la Afirmación de Ammam, hace 19 años, Colombia no ha logrado una educación básica para todos los niños y niñas. "Solo el 30 \% de los jóvenes hace la transición de la escuela o colegio a la educación superior" (Ministerio de Educación Nacional, 2016, p. 30). La educación matemática y la matemática con todos y para todos, además de ser un derecho, se vuelve una necesidad social. La ausencia de una formación matemática llega a comprometer la libertad de los ciudadanos, en cuanto a sus posibilidades de decisión y elección (Frenkel, 2015). Hay evidencias de relaciones entre un número alto de suspensiones en el colegio o detenciones por la policía, y un muy bajo desempeño en aritmética en poblaciones en edad escolar (Bynner y Parsons, 2005).

Los resultados de estudios sobre bajo desempeño académico y exclusión de poblaciones de la educación destacan factores que además de vincular situaciones de los estudiantes, involucran enfoques de evaluación (Biggs y Tang, 2007; Lafrancesco, 1996; Prodromou, 1995) y ambientes curriculares e institucionales (Reyes, Brackett, Rivers, White, y Salovey, 2012; Sinclaire, 2014interaction and communication, the course, the physical learning environment, and college facilities and services. In post-secondary education, student satisfaction is both an outcome of the learning process as well as a requirement for successful learning. Student satisfaction is linked to improved academic performance, continued learning (Sloan, n.d.).

Las metodologías para la evaluación de diseños de ambientes de aprendizaje que incorporan recursos digitales virtuales y no virtuales (Blondet y Nascimento, 2004; Fernández, Llinares y Valls, 2011; Munro y Kenny, 2008) privilegian los aspectos tecnológicos y virtuales, con poca o ninguna alusión a aspectos de la didáctica. León y López y Mota (2016) plantean la importancia de evaluaciones y validaciones que contemplen tanto lo tecnológico como lo pedagógico y didáctico. Se trata, como lo señala León (2016), de consolidar un sistema de evaluación de naturaleza didáctica para la evaluación de los diseños didácticos para formar profesores. Hasta el momento, los sistemas de evaluación dan prioridad al aprendizaje del estudiante (García-Oliveros y Monteho-Rozo, 2009), pero se ignora o se le da un papel secundario al diseño que organizó el ambiente de aprendizaje, y a las hipótesis que el profesor formador de profesores organizó para ese diseño.

1 Esta investigación está en el marco del programa nacional de Colciencias Alianza de Instituciones para el Desarrollo de la Educación y la Tecnología en Colombia-Aidetc, más específicamente en el proyecto "Desarrollo didáctico y tecnológico en escenarios didácticos para la formación de profesores que acogen la diversidad: factores para su implementación y su validación en la Universidad Distrital Francisco José de Caldas", con el código 1419-6614-44765. 
Complementariamente, la evaluación de los diseños didácticos en la formación de profesores es afectada por lo que Gil-Chaves (2016) destaca como la dificultad de establecer la relación entre los aspectos teóricos que se trabajan durante el proceso de la formación, y los problemas propios de la práctica en instituciones escolares. Esta dificultad también la reportan otros investigadores en educación matemática (Cisternas, 2011; Flores, 1998; Ponte y Chapman, 2006; Porlan, 1993).

Este artículo presenta los avances de investigación sobre la construcción de una arquitectura de validación, que permite realizar una evaluación didáctica tanto a diseños de ambientes de aprendizaje como a los modos de sustentar y organizar esos diseños (Cobb y Gravemeijer, 2008).

\section{Formación didáctica de profesores de matemáticas que acogen la diversidad}

Ante la fuerte presencia de diversidad de poblaciones en espacios escolares y sociales, es necesario que los futuros profesores de matemáticas no sean ajenos a esa situación. La formación orientada hacia el reconocimiento, la participación y la promoción de poblaciones que históricamente han sido excluidas de la educación debe ser una realidad en los currículos de formación de profesores (Association of Mathematics Teacher Educators, 2017; León et al., 2014).

La formación didáctica articula: (1) una formación como profesional de la educación matemática que integra el reconocimiento de la diversidad de poblaciones en contextos sociales, educativos y culturales, con la función de la educación matemática en el desarrollo de las sociedades; (2) una formación pedagógica, que integra las posturas éticas, ontológicas y epistemológicas que definen las orientaciones misionales y curriculares de la educación matemática en contextos educativos, y (3) experiencias en ambientes de aprendizaje interculturales y pluritecnológicos con elementos estructurantes y estructuradores de las experiencias de aprender la práctica de enseñar las matemáticas (León et al., 2014; León, Calderón, García y Martínez, 2016).

\section{Los diseños didácticos accesibles para la formación de profesores de matemáticas que acogen la diversidad}

Acoger la diversidad en educación es crear ambientes de aprendizaje accesibles, con adaptaciones que permitan la manifestación de múltiples formas de aprender, que no marginen ni discapaciten poblaciones. La accesibilidad del contenido curricular es una característica del diseño didáctico que permite a profesores y estudiantes que poseen diferentes capacidades acceder a la información presente en el diseño, usarla y transformar situaciones de aprendizaje (León et al., 2014). 


\section{Diseño Universal para el Aprendizaje}

La característica fundamental del Diseño Universal para el Aprendizaje es la de ser un marco que aborda el principal obstáculo para promover aprendices expertos en los entornos de enseñanza: los currículos inflexibles, talla-única-para-todos (Cast, 2011). Se trata de considerar el acceso a todos los aspectos del aprendizaje:

En los ambientes de aprendizaje, como colegios o universidades, la variabilidad individual es la norma y no la excepción. Cuando los currículos son diseñados para atender la media imaginaria no se tiene en cuenta la variabilidad real entre los estudiantes. Estos currículos fracasan en el intento de proporcionar a todos los estudiantes unas oportunidades justas y equitativas para aprender, ya que excluyen a aquellos con distintas capacidades, conocimientos previos y motivaciones, que no se corresponden con el criterio ilusorio de "promedio". (p. 3).

El diseño es un dispositivo para acoger diferentes condiciones de los estudiantes en el aula. Los diseños con todos plantean la satisfacción de exigencias de accesibilidad: (1) a la situación por audición, por visión, por aspectos táctiles o por aspectos perceptuales de otros órdenes; (2) al manejo de la información de la situación, bien sea por registro escrito, visual, auditivo o viso-gestual; (3) a las formas de representar y operar las relaciones y los objetos matemáticos emergentes de la información; (4) a las formas de comunicar y cooperar en el estudio de la información que propone la situación (León, Díaz y Guilombo, 2014).

El diseño didáctico para todos exige comunidades que "funcionan como comunidades de práctica, procurando activamente la consecución de una empresa, negociando sus formas de participación y desarrollando sus propias historias" (Wenger, 2001, p. 273). Las siguientes dimensiones duales son propias de los diseños didácticos accesibles, en ambientes de comunidades de práctica:

» Participación/cosificación: en las prácticas de aprendizaje se participa haciendo cosas con instrumentos adecuados a la práctica, y las cosas que se hacen modifican los tipos de participación de quienes las producen.

» Local/global: la práctica local de un estudiante, con un caso particular de un campo de conocimiento, debe relacionarse con la práctica global de todo el grupo social que indaga por un fenómeno de conocimiento propio del campo estudiado localmente. La relación dinámica local-global permite la evolución mutua.

» Negociabilidad de significados/identificación: los modos de significar y los significados de la práctica los provee la comunidad, y los participantes se identifican en ellos. Sin embargo, las personas 
no aprenden esos modos y significados de manera neutra, sino que los modifican y adaptan.

» Emergencia de aprendizaje/diseños flexibles: aunque la propuesta de aprendizaje se diseña, el aprendizaje es una respuesta de los aprendices frente a ese diseño. Así que, durante el aprendizaje, emergerán situaciones, y el diseño debe tener las condiciones para permitirlas (León et al., 2013).

La ciencia del diseño aplicada a la educación plantea que la calidad del aprendizaje acumulado es el efecto de las características de los estudiantes, los procesos de alineación constructivos y los ambientes de enseñanza y aprendizaje que articulan los dos aspectos anteriores (Laurillard, 2012).

Cuando en la didáctica de la matemática se incorpora el reconocimiento de la diversidad de poblaciones y la exigencias por diseños accesibles se inicia el proceso de reconceptualizar objetos didácticos y de articular marcos de referencia, para hacer visibles aspectos que no han sido considerados prioritarios por muchos años en la didáctica de las matemáticas y que, posiblemente, son la causa de la marginación de la educación de muchas poblaciones.

\section{El diseño de un Curso Fuente para la formación didáctica de profesores}

Los formadores de estudiantes para profesores de matemáticas son docentes universitarios con autonomía y libertad de cátedra. Su idoneidad didáctica se vincula tanto con la competencia de diseño, gestión y evaluación de la enseñanza y el aprendizaje, como con la calidad del aprendizaje de sus estudiantes (Godino, 2009; 2014). En consecuencia, un formador de profesores realiza prácticas de creación, reinvención, reconstrucción e innovación en la formación profesional de sus estudiantes (León et al., 2014). El curso como recurso para un formador de estudiantes para profesor es una fuente para las prácticas mencionadas; se espera que el diseño del curso se incorpore en los demás que realice el formador.

La organización del curso destaca como componentes estructurales: los institucionales, los intencionales, los contextuales, los pedagógicos, los didácticos, los instrumentales y los evaluativos. Los fundamentos para el diseño instruccional del curso se presentan en la figura 1.

El diseño instruccional "es el proceso sistemático que conduce a la creación de sistemas instruccionales, mientras que el desarrollo instruccional es el proceso de implementar dicho sistema o plan e incluye su evaluación y mantenimiento" (Díaz-Barriga, 2006, p. 5) (véase la figura 2). 


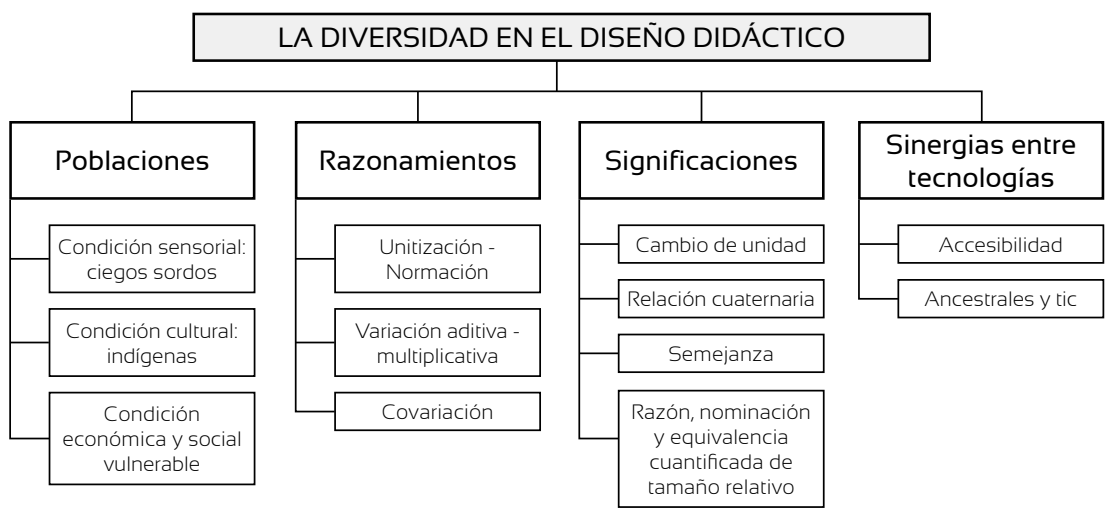

Figura 1. Fundamentos para un curso sobre didáctica de la proporcionalidad. Fuente: elaboración del autor.

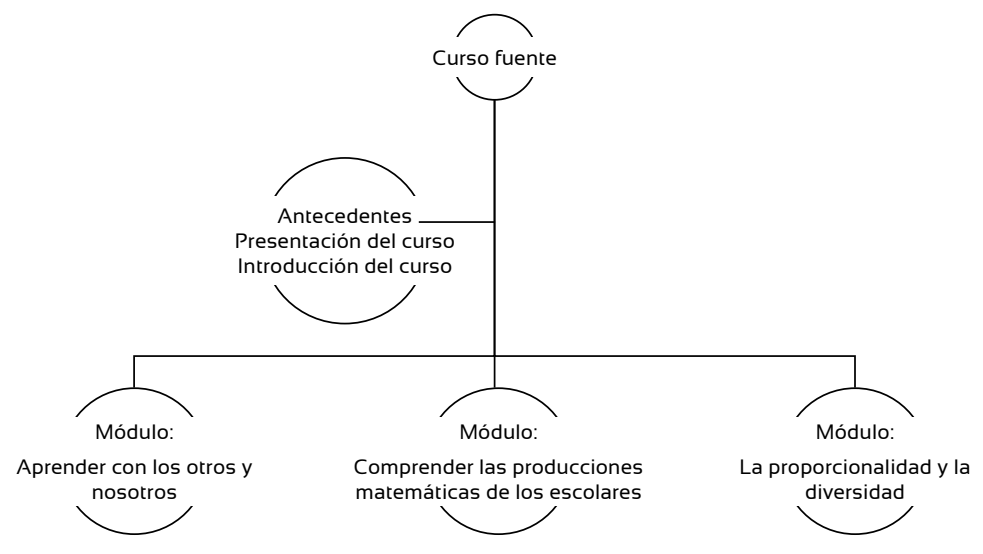

Figura 2. Estructura curso fuente.

Fuente: elaboración del autor.

La modalidad del curso semipresencial, o B-Learning (Fernández et al., 2011), se caracteriza como una experiencia que combina el conjunto de interacciones cara a cara con interacciones en línea (virtuales). Esta modalidad exige la elección de una plataforma para la interacción en línea. ATutor es un sistema de gestión de contenidos de aprendizaje (LCMS) de código abierto, basado en la web y diseñado con el objetivo de lograr accesibilidad y adaptabilidad. ATutor es el primer LCMS completamente conforme a las especificaciones de la accesibilidad de w3C-WAI (2016) WCAG 1.0 en el nivel de AA+. Permite el acceso a todos los estudiantes potenciales, instructores y administradores, incluyendo a quienes cuentan con problemas de acceso usando tecnologías asistidas. 


\section{Objetos virtuales de aprendizaje accesibles}

Con los objetos virtuales de aprendizaje (OvA) incorporados en el curso fuente se busca identificar el reconocimiento, la participación y la promoción de la diversidad de poblaciones y su acogimiento en la formación de profesores. El diseño incorpora una estructura que contiene orientaciones técnicas, pedagógicas y consideraciones para el trabajo del estudiante en tres momentos: momentos de experiencias individuales, momentos de experiencias grupales y momentos de evaluación.

Los ova no solo cumplen con los requerimientos de portabilidad, granularidad y mutabilidad de un objeto virtual, sino que la misma estructura impone un trabajo que involucra la generación de un ambiente de aprendizaje con condiciones de accesibilidad (García-García, 2006).

Los criterios de accesibilidad y portabilidad necesarios para la creación de los ova se aplican en la selección del Exelearning; de todas las herramientas que este dota para la construcción de los objetos, se destacan las que permiten hacer visible el tratamiento de la accesibilidad. Por ejemplo, videos en lengua de señas, videos con audio y subtítulos para la población sorda, uso de herramientas de ampliación de imagen, textos alternativos y demás consideraciones para poder llegar a diferentes poblaciones.

El uso de textos alternativos en los ova se ilustra con la imagen 1, que muestra el video en lengua de señas, como texto alternativo para poblaciones sordas. En la imagen 2 se presenta la organización del contenido del curso en pestañas, que pueden desplegarse según el interés del estudiante o del profesor.

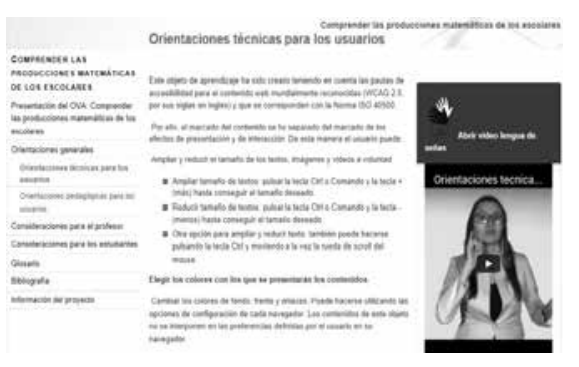

Imagen 1. Presentación de los requerimientos técnicos para utilizar el ova, usando además video en lengua de señas. Fuente: autor.

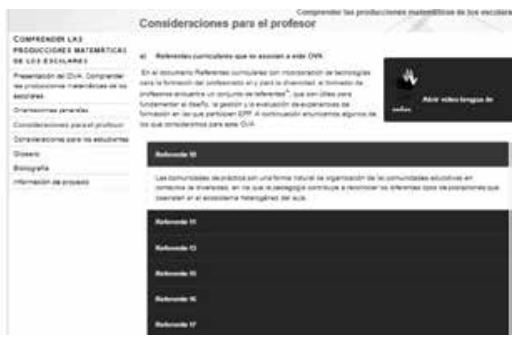

Imagen 2. Consideraciones teóricas para el docente, usando además video en lengua de señas. Fuente: autor. 
Las imágenes 3 y 4 muestran el uso del video, del texto y de las herramientas de ampliación de imagen. Señalan una forma de presentar la misma situación, pero dispuesta para una población de baja visión.

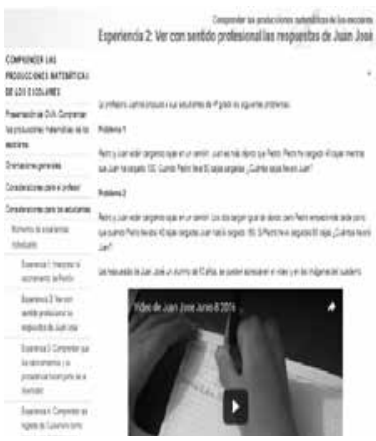

Imagen 3. Video de la forma de resolución de un estudiante presentado en video. Fuente: autor.

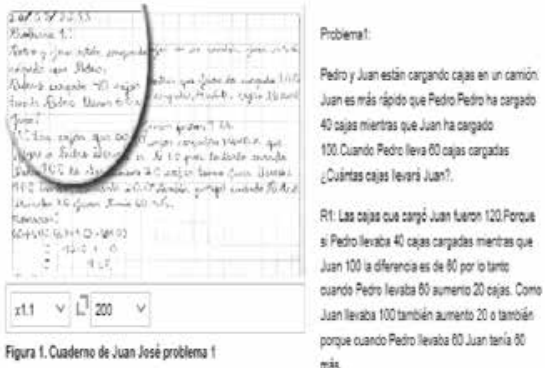

Imagen 4. Uso de lupa para ampliar el texto, además el texto está transcrito para lector de pantalla. Fuente: autor.

La imagen 5 muestra un video de dos estudiantes en condición de discapacidad visual manipulando las regletas de Cuisenaire, y la imagen 6 muestra el uso de la lupa para realzar el tejido de la artesanía. Dichos videos fueron adaptados con audiodescripción y subtitulación, lo que facilita el uso por mayor variedad de poblaciones. En la segunda figura se usa la ampliación de imagen para detectar patrones y regularidades en un tejido.

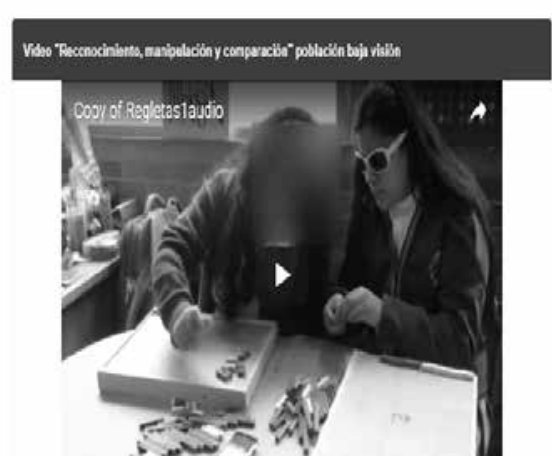

Imagen 5. Videos con niñas de baja visión y baja audición. Fuente: autor

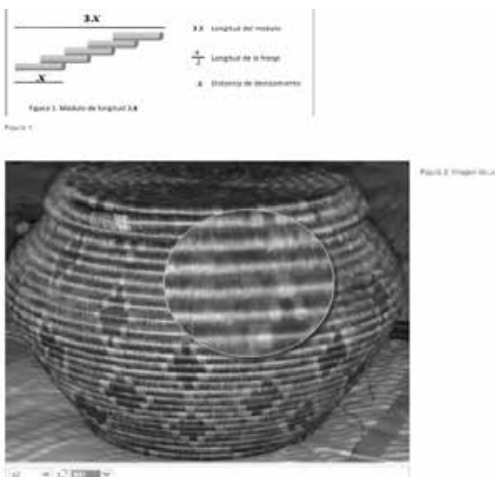

Imagen 6. Reconocimiento de patrones geométricos en tejido. Fuente: autor.

En este sentido, la didáctica del acogimiento de la diversidad en educación matemática actúa como ciencia del diseño: En esta investigación las siguientes palabras de Laurillard (2012) "enseñanza es [...] parecida a 
una ciencia del diseño porque utiliza lo que se conoce sobre la enseñanza para alcanzar la meta de aprendizaje del estudiante, y utiliza la puesta en escena de sus diseños para seguir mejorándolos" (p. 1, traducción propia) son referente para la elaboración de los diseños didácticos.

\section{Metodología}

El diseño de una arquitectura como herramienta metodológica para validar, desde una perspectiva didáctica, diseños didácticos para la formación de profesores, se estructura a partir de los siguientes procesos que provienen de metodologías de investigación con aplicación en educación:

» Refinamiento de los diseños de ambientes didácticos y ova para formar profesores de matemáticas que acogen la diversidad (León et al., 2016). Se procede con herramientas de diseño de la ciencia del diseño.

» Refinamiento de la formulación de hipótesis de investigación sobre tipos de relaciones didácticas que son de necesaria consideración para formar profesores de matemáticas que no marginen poblaciones. Se procede con herramientas de la metodología de los experimentos de enseñanza.

» Refinamiento de estructuras de organización de investigadores en educación matemática que conforman comunidades de práctica de investigadores que fortalecen los valores de la investigación acción promovidos por Fals Borda (1978). Se procede con herramientas de la metodología de comunidad de práctica.

\section{La ciencia del diseño}

Esta investigación se ubica en la ciencia del diseño (Dresh, Pacheco y Valle, 2015), por cuanto tiene como propósito y como meta de investigación no solo explorar, describir y explicar fenómenos de enseñanza y aprendizaje de las matemáticas, sino diseñar un ambiente de aprendizaje accesible, para:

... explicar por qué el diseño instruccional propuesto funciona, y sugerir formas con las cuales puede ser adaptado a nuevas circunstancias. Se incluye y refleja un compromiso para entender las relaciones existentes entre teoría educativa, práctica e instrumentos. (Molina, Castro, Molina y Castro, 2011, p. 76).

De la estructura macro de la investigación de diseño se retoman los estudios de Dresh, Pacheco y Valle (2015) sobre procesos y fases de este tipo de investigación, y se aplica en el diseño de ambientes de aprendizaje y OVA, para formar profesores de matemáticas que acogen la diversidad, con un diseño para evaluación (Göbel y Cronholm, 2012). 


\section{Experimentos de enseñanza}

La evolución en escenarios naturales de los diseños didácticos articula la arquitectura de validación los experimentos de enseñanza (Brown, 1992; Clements y Sarama, 2009; Gómez y Lupiañez, 2007; Molina y Castro, 2006; Simon y Tzur, 2004). El experimento de enseñanza se desarrolla en ambientes en los cuales los participantes suelen ser: un docente, uno o más estudiantes y un investigador. La duración del experimento es variable, y en el ambiente pueden participar pequeños grupos en el aula de clase (León, Díaz y Guilombo, 2014). Las fases del desarrollo del experimento de enseñanza que se incorporan a la arquitectura se retoman de las propuestas de Cobb y Gravemeijer (2008).

\section{Las comunidades de práctica}

Según esta perspectiva, es posible asumir la formación de profesores como el ingreso paulatino a una comunidad de práctica (Wenger, 2001) de profesores, en la que el estudiante para profesor aprende la práctica de enseñar que realizan sus miembros. En la comunidad existen y se diferencian niveles de experticia y de participación; los aprendices evolucionan en la práctica desde una participación periférica legítima, mientras que los miembros expertos se involucran de manera plena, se consideran miembros de pleno derecho, incluso constituyen trayectorias paradigmáticas que se convierten en referencia de llegar a ser (Wenger, 2001).

\section{Caracterización de la arquitectura de validación}

En la arquitectura, un ámbito se concibe como una zona de cuestiones y problemas ligados a la investigación, que pertenecen al refinamiento de la práctica de formación de profesores de matemáticas que acogen la diversidad. La arquitectura se estructura con tres ámbitos diferenciados, pero completamente relacionados en el desarrollo de una práctica didáctica que vincula procesos de enseñanza y aprendizaje:

» Ámbito de la realización y del refinamiento de la práctica como experiencia de llegar a ser profesor. Es el ámbito en el que ocurre la evolución del diseño didáctico en escenarios naturales.

» Ámbito de gestión de la investigación y del refinamiento de la práctica como experiencia de ser miembro de pleno derecho. Es el ámbito en el que organizan formas de observar y hacer seguimiento a la evolución de los diseños.

» Ámbito de la reflexión metadidáctica y del refinamiento de la práctica como experiencia de constelación. Es el ámbito en el que se consolida la práctica reflexiva de las comunidades, para lograr identificar los resultados de la evolución del diseño. 
» Los tres ámbitos para el refinamiento de la práctica didáctica se caracterizan por ser escenarios para el desempeño de roles apropiados para las prácticas y la emergencia de los procesos que le otorgan idoneidad a la práctica. Finalmente, la arquitectura se complementa con lo que se denomina los corredores de la arquitectura, que son las formas y medios para la circulación de información entre los tres ámbitos, como se muestra en la imagen 7.

\section{ARQUITECTURA DE VALIDACIÓN DE DISEÑOS DIDÁCTICOS}

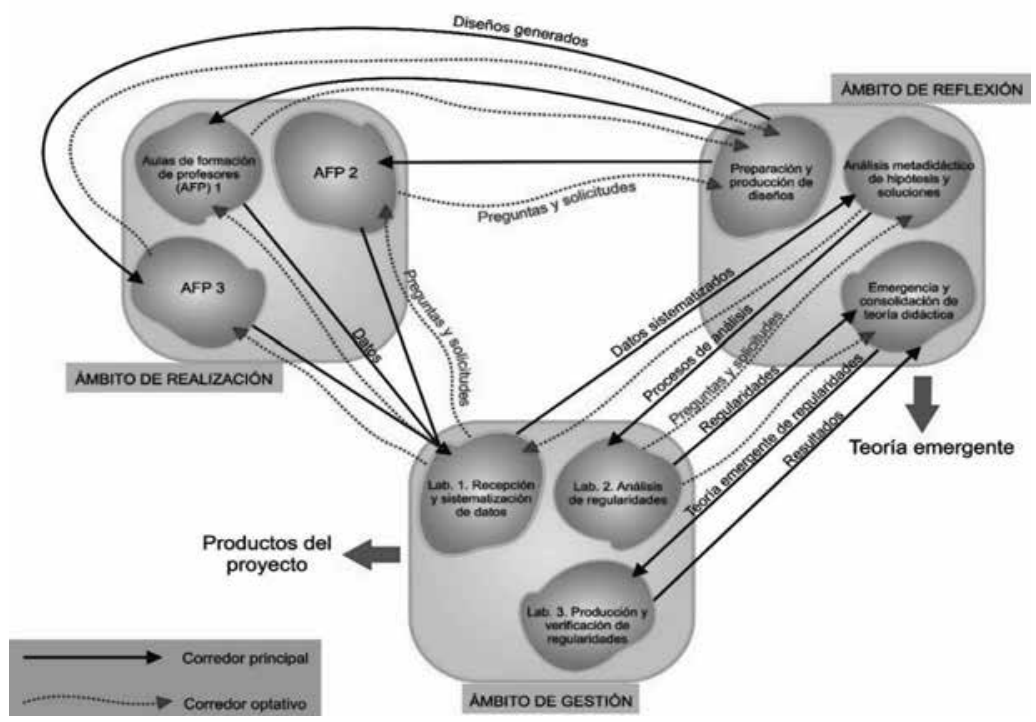

Imagen 7. Arquitectura para la validación de diseños didácticos en escenarios naturales.

Fuente: elaboración del autor.

Ámbito de la realización y del refinamiento de la práctica didáctica como experiencia de llegar a ser profesor

En este ámbito se trata de indagar cómo el formador de profesores y los estudiantes para profesores se identifican a sí mismos como competentes en la práctica didáctica, con sentido de pertenencia a una comunidad que los convoca para realizarla. Es vivir la experiencia de ser profesor de matemáticas para el acogimiento de la diversidad en el aula de esta materia.

Escenarios naturales: Se conciben como el escenario natural de formación de profesores de matemáticas, y se constituye materialmente por las aulas de formación; aulas que pueden ser físicas, virtuales o mixtas y, además, caracterizarse por su presencia en los programas o propuestas de formación que se realizan en el aula (Calderón y León, 2015). 


\section{Ámbito de gestión de la investigación y del refinamiento de la práctica como experiencia de ser miembro de pleno derecho}

En este ámbito, se trata de indagar y comprender los ambientes de aprendizaje, según dinámicas de procesamiento y sistematización de datos provenientes de los escenarios naturales del ámbito de realización, a partir de los instrumentos de observación y análisis que dispone y organiza la arquitectura para este fin. En este escenario se revisan las hipótesis de los investigadores. Este ámbito estructura la investigación y exige esa estructura (fases, categorías, instrumentos de recolección de datos, técnicas de análisis, etc.). Su finalidad es la gestión de la investigación.

Los escenarios naturales del ámbito de la realización del diseño didáctico. En este ámbito se configuran cuatro escenarios definidos por su función en la investigación como laboratorios²:

» Laboratorio de recepción y codificación de información (proveniente del ámbito de realización del diseño)

» Laboratorio de sistematización de la información

» Laboratorio de análisis de regularidades

" Laboratorio de producción de resultados

Corredores: En su relación con otros ámbitos, en la dinámica de la investigación, el ámbito de gestión de la investigación establece los siguientes corredores:

» Plataformas para organización de datos provenientes del ámbito de realización

» Mesas de trabajo, entre investigadores y profesores formadores, para el análisis de datos de observación y devolución de resultados a los escenarios naturales del ámbito de realización

» Plataformas de gestión de los investigadores para el debate y la comunicación de resultados, vinculados a los datos sistematizados, al análisis de regularidades y posibles surgimientos de categorías emergentes

\section{Ámbito de la reflexión metadidáctica y del refinamiento de la práctica como experiencia de constelación}

Para el proyecto de investigación, este ámbito recoge la generación de hipótesis didácticas. En este ámbito se consolida una didáctica como la téchnē de la educación matemática. Se caracteriza por ser el espacio de la investigación y de la construcción metadidáctica, a partir de la

2 Espacio de organización, análisis, reflexión y reconfiguración por parte de la comunidad cam de investigadores. 
generación de conjeturas didácticas y la emergencia de teorías en los otros ámbitos, para producir el diseño. En la relación entre los tres ámbitos, en este se gesta la semilla del diseño didáctico.

Escenarios. En el desarrollo del ámbito de reflexión emergen tres escenarios:

» Escenario de revisión de hipótesis y soluciones, cuyos procesos están definidos por ciclos de diseño: revisión de hipótesis, construcción de soluciones para gestar y producir el diseño didáctico.

» Escenario de convergencia y consolidación de teoría didáctica, cuyos ciclos son: de emergencia de teoría y de consolidación de teoría didáctica.

» Escenarios naturales o de evolución de los experimentos de enseñanza. Los ciclos producidos en esta evolución son intermediarios de los ciclos de revisión de hipótesis y soluciones y de los de emergencia de teoría y consolidación didáctica.

Corredores. El ámbito de reflexión establece corredores hacia:

El ámbito de realización del diseño: recibe preguntas sobre el diseño, los Ambientes Virtuales de aprendizaje y los ova. Así, el ámbito de realización de diseño no se separa de este ámbito de reflexión, en la validación.

El ámbito de gestión de la investigación: recibe sistematización de datos e identifica en dónde hay emergencia teórica y devuelve al ámbito de gestión de la investigación para consolidar resultados.

\section{Las categorías didácticas para la valoración de los diseños, y los instrumentos de observación}

Las categorías de la investigación que activan la dinámica de la arquitectura provienen de las hipótesis didácticas que organizaron el Curso Fuente y fundamentaron el diseño de los ova incorporados en el curso. La tabla 1 presenta categorías y descriptores para la observación.

Tabla 1. Categorías y descriptores del instrumento de observación del curso. Fuente: elaboración del autor.

\begin{tabular}{|l|l|}
\hline \multicolumn{1}{|c|}{ Categorías } & \multicolumn{1}{c|}{ Descriptores } \\
\hline \multirow{4}{*}{ Acogimiento a la diversidad } & Reconoce diversidad de poblaciones \\
\cline { 2 - 2 } & Participa con diversidad de poblaciones \\
\cline { 2 - 2 } & Promueve el aprendizaje entre poblaciones diversas \\
\hline \multirow{4}{*}{$\begin{array}{c}\text { Conformación de la comu- } \\
\text { nidad }\end{array}$} & Interacción en pequeños grupos \\
\cline { 2 - 2 } & Interacción en plenarias \\
\cline { 2 - 2 } & Interacción en comunidades globales \\
\hline
\end{tabular}




\begin{tabular}{|l|l|}
\hline \multicolumn{1}{|c|}{ Categorías } & \multicolumn{1}{c|}{ Descriptores } \\
\hline \multirow{y}{*}{$\begin{array}{l}\text { Accesibilidad a procesos } \\
\text { de formación }\end{array}$} & $\begin{array}{l}\text { A tipos de representación de los contenidos del aprendizaje } \\
\text { (en lengua natural, en registros figurales, registros numéri- } \\
\text { cos, registros algebraicos, entre otros) }\end{array}$ \\
\cline { 2 - 2 } & $\begin{array}{l}\text { A tipos de razonamiento en las experiencias de aprendizaje } \\
\text { (inductivo, deductivo, abductivo) }\end{array}$ \\
\cline { 2 - 2 } & $\begin{array}{l}\text { A formas de comunicación (oral, escrita, gestual, monolingüe, } \\
\text { bilingüe) }\end{array}$ \\
\cline { 2 - 2 } & $\begin{array}{l}\text { A procesos de constitución de identidad de ser profesor } \\
\text { (elaboración de diseños para poblaciones diversas, inter- } \\
\text { pretación de producciones matemáticas de estudiantes de } \\
\text { poblaciones diversas, evaluación de diseños didácticos para } \\
\text { poblaciones diversas) }\end{array}$ \\
\hline
\end{tabular}

Fuente: elaboración propia.

El instrumento de observación es diligenciado por el profesor, el observador y los estudiantes participantes en el curso, en cada sesión de clases. .

La tabla 2 presenta categorías y descriptores para el seguimiento del desarrollo del curso con incorporación de los ova.

Tabla 2. Categorías y descriptores del instrumento de evaluación del curso. Fuente: elaboración del autor.

\begin{tabular}{|c|c|}
\hline \multirow{5}{*}{$\begin{array}{l}\text { Institucional } \\
\text { Se pretende dar cuenta de } \\
\text { los recursos y las condi- } \\
\text { ciones que posee la insti- } \\
\text { tución para llevar a cabo el } \\
\text { desarrollo del curso. } \\
\text { (5 ítems) }\end{array}$} & En la institución existen procesos para registrar el curso. \\
\hline & $\begin{array}{l}\text { En la institución existen procesos y espacios para publicar el } \\
\text { programa del curso. }\end{array}$ \\
\hline & El curso forma parte del plan de estudios de la carrera. \\
\hline & $\begin{array}{l}\text { Las condiciones institucionales existentes para el uso de tic } \\
\text { en los cursos (disponibilidad de hardware y software) son las } \\
\text { que se requieren. }\end{array}$ \\
\hline & $\begin{array}{l}\text { Las condiciones institucionales de la planta fisica (ventila- } \\
\text { ción, iluminación, accesibilidad, visibilidad, acústica, entre } \\
\text { otras) son adecuadas para el curso. }\end{array}$ \\
\hline \multirow{5}{*}{$\begin{array}{l}\text { Intencional: } \\
\text { Se pretende evaluar los } \\
\text { objetivos o propósitos del } \\
\text { curso propuesto. } \\
\text { (5 ítems) }\end{array}$} & Los objetivos o propósitos del curso son explícitos. \\
\hline & $\begin{array}{l}\text { Los objetivos o propósitos del curso propenden por la for- } \\
\text { mación de profesores de matemáticas. }\end{array}$ \\
\hline & $\begin{array}{l}\text { Los objetivos o propósitos del curso propenden por el } \\
\text { desarrollo de competencias para reconocer la diversidad de } \\
\text { poblaciones en el aula de matemáticas. }\end{array}$ \\
\hline & $\begin{array}{l}\text { Los objetivos o propósitos del curso propenden por el desa- } \\
\text { rrollo de competencias para crear contenidos accesibles. }\end{array}$ \\
\hline & $\begin{array}{l}\text { Los objetivos o propósitos del curso propenden por el } \\
\text { desarrollo de competencias para conformar comunidades } \\
\text { de práctica. }\end{array}$ \\
\hline
\end{tabular}




\section{Contextual}

Se pregunta sobre la infraestructura física y virtual con la cual se desarrolló el curso.

(3 ítems)

\section{Pedagógica}

Se pregunta sobre la formación de profesores para poblaciones diversas.

(1 ítem)

\section{Didáctica}

Indaga sobre los procesos de aprendizaje y de enseñanza, los contenidos, los recursos dados en el curso. (8 ítems)

\section{Instrumenta}

Hace referencia a los diferentes instrumentos utilizados durante el curso, su selección, diseño y relaciones con el entorno. (8 ítems)
La infraestructura física de la institución, para entornos no virtuales, es la requerida para los objetivos o propósitos del curso

La infraestructura TIC de la institución, para entornos virtuales (servidores, plataforma, soporte físico, soporte técnico, conexiones, software, ...), es la requerida para los objetivos o propósitos del curso

Las infraestructuras para entornos virtuales y no virtuales se vinculan para alcanzar los objetivos o propósitos del curso.

El curso ofrece condiciones para la formación de profesores de matemáticas que no marginan poblaciones.

Los procesos de enseñanza promueven el desarrollo de trayectorias de aprendizaje de la didáctica de la matemática.

Los procesos de aprendizaje desarrollan la trayectoria propuesta por el profesor.

Las experiencias de aprendizaje del curso se vinculan a niveles de trayectorias de aprendizaje.

Los contenidos y recursos de las experiencias de aprendizaje son accesibles para poblaciones ciegas, sordas, oyentes, videntes y desplazadas.

Los ova del curso fuente fueron usados para incorporar experiencias de aprendizaje en este curso.

Las experiencias de aprendizaje promueven la interacción entre diferentes poblaciones.

Las experiencias de aprendizaje promueven conformación de comunidades de práctica entre profesor y estudiantes.

El curso pone en acción elementos de una didáctica de la matemática para todos

Para el desarrollo adecuado del curso se requieren nuevos recursos tecnológicos en la institución.

Los recursos de la infraestructura TIC son necesarios para la evolución del curso.

Los recursos de la infraestructura física son necesarios para la evolución del curso.

La selección o el diseño de los recursos se hacen con criterios de accesibilidad para los estudiantes.

Las relaciones entre los entornos tecnológicos (virtuales y no virtuales) favorecen las experiencias de aprendizaje.

El curso incluye recursos para hacer seguimiento del aprendizaje de los estudiantes.

La plataforma ATutor es un entorno tecnológico adecuado para el curso

Se accedió a los ova en el repositorio Alter-Nativa 


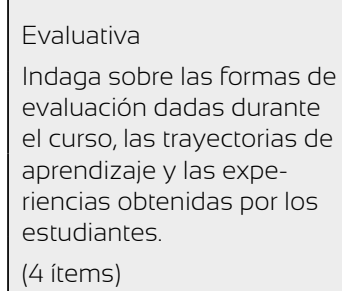

Las experiencias y los recursos empleados permitieron lograr los objetivos o propósitos del curso

Las relaciones entre los entornos físicos y virtuales permitieron la evolución de las experiencias de aprendizaje.

Hay evidencia de trayectorias de aprendizaje de la didáctica de la matemática para todos.

Los estudiantes reconocen su aprendizaje como un efecto de su participación en el curso.

Fuente: elaboración propia.

El instrumento de evaluación lo diligencian el profesor, el observador y los estudiantes participantes en el curso, en la mitad del proceso de su implementación y al finalizarlo. Sus valoraciones son de tipo cuantitativo.

\section{Espacio de formación de profesores de matemáticas en el que se implementó la arquitectura}

La arquitectura se implementó en tres escenarios naturales de formación de profesores, dos en universidad pública y uno en universidad privada. Se presentan los resultados de la implementación del curso Práctica Intermedia II, cuyo énfasis es en recursos didácticos, que forma parte del plan de estudios de la Licenciatura en Educación Básica con Énfasis en Matemáticas. La evolución del diseño la hace un profesor, un observador y once estudiantes del curso.
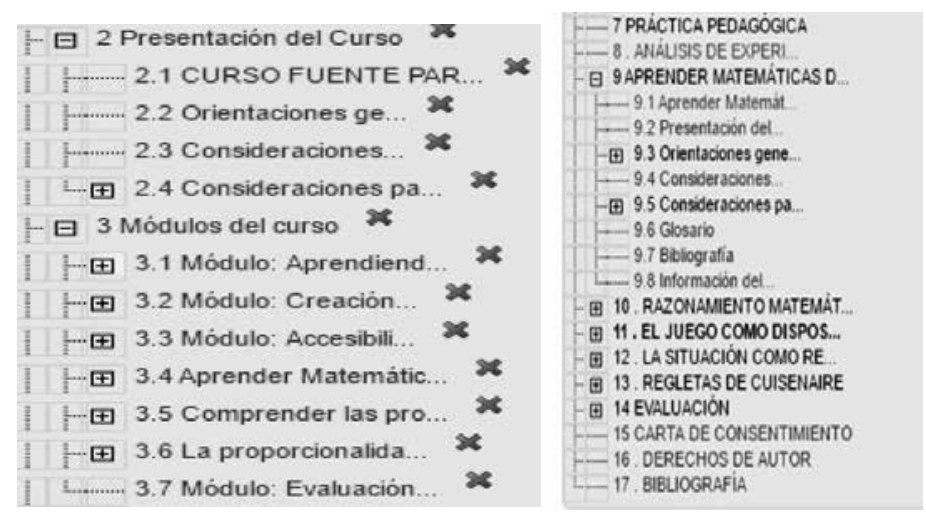

Imagen 8. Planteamiento del curso en el formato de ATutor. (Izquierda curso fuente, derecha curso Práctica Intermedia II)

La imagen 8 muestra la relación entre la estructura del curso fuente y el diseño realizado por el profesor del curso Práctica Intermedia II, en cuyos objetivos se retoma el trabajo de accesibilidad y acogimiento de poblaciones dispuesto en el fuente, manteniendo las particularidades de su intención formativa sobre práctica y recursos didácticos. 


\section{Resultados}

Los resultados que se presentan a continuación corresponden a dos de los tres ámbitos para el refinamiento de la práctica didáctica: el de la realización y el de la gestión, y se vinculan a la validación de la hipótesis: formar profesores de matemáticas que acogen la diversidad de poblaciones es generar diseños accesibles.

En el ámbito de la realización y del refinamiento de la práctica didáctica como experiencia de llegar a ser profesor se desarrolló el curso Práctica II Intermedia, el escenario natural de las clases bajo la orientación del profesor, diseñador del curso, los estudiantes y la participación de un observador. En el ámbito de gestión de la investigación y del refinamiento de la práctica como experiencia se hace la indagación y comprensión del desarrollo y seguimiento de la evolución del experimento de enseñanza. Los escenarios naturales correspondientes a este ámbito son: el laboratorio de recepción y codificación de información, que recoge elementos provenientes de los tres actores para consolidar el diseño del ambiente didáctico, desde el profesor (que propone), el observador (que analiza lo sucedido) y los estudiantes. El laboratorio de sistematización de la información, que utiliza los diferentes corredores (plataformas, mesas de trabajo, plataformas de gestión de los investigadores), procesa los datos de los instrumentos de observación (se diligencian en cada sesión) y de evaluación (se diligencian en dos momentos, mitad y final del curso) por cada uno de los actores participantes en el curso. El laboratorio de análisis de regularidades también usa los diferentes corredores, estudia, comprende y cuestiona las regularidades, relaciones y diferencias entre las valoraciones de los diferentes actores en las sesiones de las clases y que han diligenciado los instrumentos.

La tabla 3 presenta la sistematización de los datos obtenidos del instrumento de observación de los descriptores 7 (Accesibilidad a tipos de representación de los contenidos del aprendizaje) y 10 (Accesibilidad a procesos de constitución de identidad de ser profesor).

Las descripciones de cada uno de los actores participantes en la evolución del experimento evidencian el surgimiento de las relaciones entre accesibilidad, diversidad y aprendizaje de las matemáticas como un elemento de tematización de los diferentes actores. 
Tabla 3: Sistematización de los descriptores 7 y 10 del instrumento de observación.

\section{SISTEMATIZACIÓN DE DATOS PARA AMBIENTES DE APRENDIZAJE}

Descriptor n. ${ }^{\circ}$. Accesibilidad a tipos de representación de los contenidos del aprendizaje (en lengua natural, en registros figúrales, registros numéricos, registros algebraicos, entre otros)

\begin{tabular}{|l|l|l|l|}
\hline Profesor & Observador & Estudiante & $\begin{array}{l}\text { Evolución del } \\
\text { experimento }\end{array}$ \\
\hline $\begin{array}{l}\text { Los contenidos } \\
\text { de aprendizaje se } \\
\text { presentan en texto, } \\
\text { lengua de señas, } \\
\begin{array}{l}\text { video con subtítulos, } \\
\text { lenguaje oral. }\end{array}\end{array}$ & $\begin{array}{l}\text { Las actividades } \\
\text { los lleva a la } \\
\text { utilización de } \\
\text { imágenes, foto- } \\
\text { grafías, lenguaje } \\
\text { oral y escrito. }\end{array}$ & $\begin{array}{l}\text { corno no virtual la representación } \\
\text { de los contenidos de aprendizaje } \\
\text { se hace explícita mediante el } \\
\text { lenguaje natural a manera de } \\
\text { socialización. Este ítem se ve } \\
\text { reflejado en las producciones he- } \\
\text { chas en Word, como reflexiones } \\
\text { de las conclusiones grupales, y } \\
\text { además en las presentaciones de } \\
\text { PowerPoint teniendo en cuenta } \\
\text { que sean accesibles. }\end{array}$ & $\begin{array}{l}\text { Se reconocen } \\
\text { diferentes formas } \\
\text { de representa- } \\
\text { ción. }\end{array}$ \\
\hline
\end{tabular}

Descriptor n. ${ }^{10}$. Accesibilidad a procesos de constitución de identidad de ser profesor (elaboración de diseños para poblaciones diversas, interpretación de producciones de estudiantes de poblaciones diversas, evaluación de diseños para poblaciones diversas)

\begin{tabular}{|c|c|c|c|}
\hline Profesor & Observador & Estudiante & $\begin{array}{l}\text { Evolución del } \\
\text { experimento }\end{array}$ \\
\hline $\begin{array}{l}\text { Los estudiantes } \\
\text { se informan sobre } \\
\text { la construcción de } \\
\text { documentos accesi- } \\
\text { bles y se reflexiona } \\
\text { sobre la importancia } \\
\text { de los mismos para } \\
\text { su formación. Los } \\
\text { estudiantes, en su } \\
\text { papel de profesores, } \\
\text { toman en considera- } \\
\text { ción la presencia de } \\
\text { estudiantes ciegos } \\
\text { y sordos para crear } \\
\text { presentaciones } \\
\text { accesibles. }\end{array}$ & $\begin{array}{l}\text { Las actividades } \\
\text { están orienta- } \\
\text { das a realizar } \\
\text { la reflexión } \\
\text { sobre reconocer } \\
\text { y promover la } \\
\text { diversidad. }\end{array}$ & $\begin{array}{l}\text { Considero que este es el punto } \\
\text { clave de la sesión ya que siempre } \\
\text { se gira en torno a la idea de ser } \\
\text { un profesor que genere inclusión, } \\
\text { reconociendo no solo los tipos } \\
\text { de discapacidad, sino también los } \\
\text { diferentes contextos sociocultu- } \\
\text { rales y los talentos excepciona- } \\
\text { les, lo cual es enriquecedor en } \\
\text { nuestro proceso de profesores } \\
\text { en formación. En este espacio de } \\
\text { interacción virtual ha sido de gran } \\
\text { ayuda (bien sea por los docu- } \\
\text { mentos propuestos sobre las } \\
\text { características que se deben tener } \\
\text { en cuenta al pensar en materiales } \\
\text { accesibles), porque gracias a ellos } \\
\text { hemos podido ampliar mucho } \\
\text { más nuestra mente, sobre todo } \\
\text { cuando se manejan algunas } \\
\text { herramientas de Office que usual- } \\
\text { mente uno no conoce, ni maneja } \\
\text { a la hora de hacer presentaciones, } \\
\text { y que considero que gracias a } \\
\text { socializaciones anteriores, y a } \\
\text { las diapositivas hechas en este } \\
\text { ejercicio están mucho mejores, y } \\
\text { teniendo en cuenta consideracio- } \\
\text { nes de accesibilidad. }\end{array}$ & $\begin{array}{l} \\
\text { Se evidencia que } \\
\text { las actividades } \\
\text { del ova posibili- } \\
\text { tan procesos de } \\
\text { constitución de } \\
\text { la identidad del } \\
\text { profesor y del } \\
\text { reconocimiento } \\
\text { de la diversidad. }\end{array}$ \\
\hline
\end{tabular}

Fuente: elaboración propia. 
Los resultados obtenidos al consolidar las valoraciones asignadas por el profesor, el observador y los estudiantes participantes del curso para cada una de las siete categorías se presentan en la imagen 9.

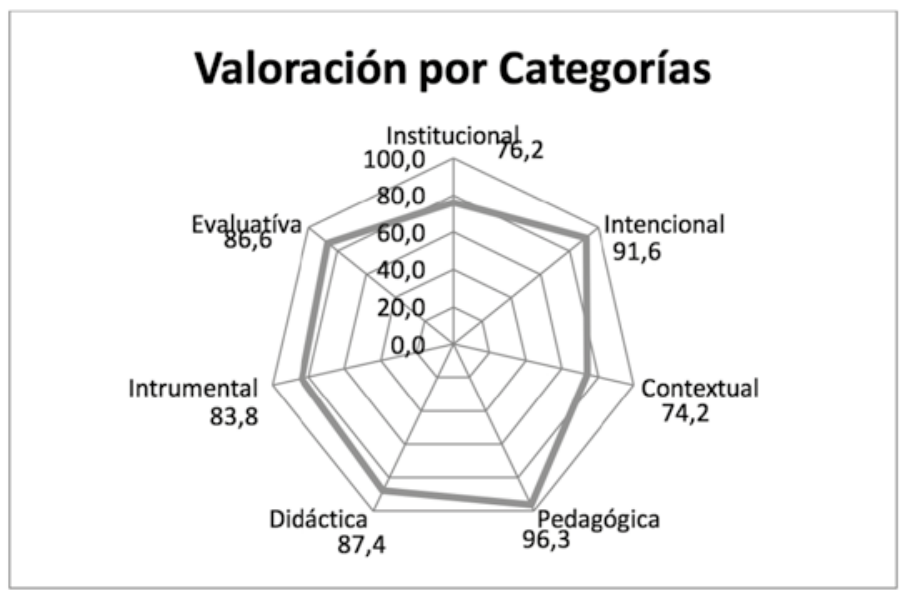

Imagen 9. Diagrama radial de los resultados generales del instrumento de evaluación. Fuente: elaboración del autor.

La categoría Pedagógica obtuvo mayor valoración con un puntaje de 96,3, donde el menor valor es 0 y el mayor es 100 , lo que muestra la pertinencia del curso como indispensable en la formación de profesores que acogen poblaciones. En la gráfica 1 se relacionan los valores altos y bajos de cada categoría con sus respectivos descriptores:

Descriptores con valoraciones más altas (1 Cuartil)

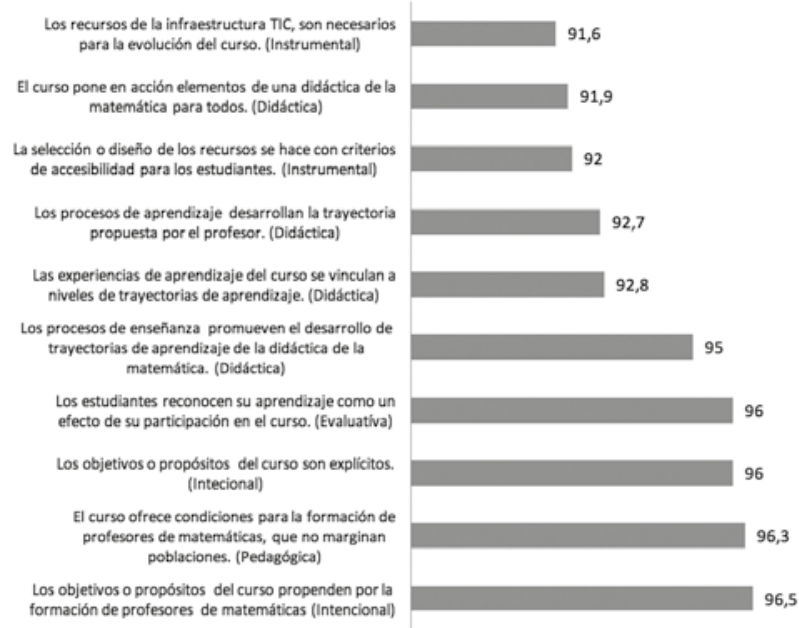


Se observa que la categoría Didáctica tiene más descriptores con valoraciones altas, se han registrado 4 de los 10 dentro del primer cuartil.

Las valoraciones más altas corresponden a: los objetivos o propósitos del curso en la formación de profesores (96,5 y 96); las condiciones de no marginación de poblaciones (96,3); el reconocimiento de su aprendizaje como estudiantes (96); las trayectorias de aprendizaje (95 y 92,8); el diseño de recursos accesibles (92). Corroboran lo expresado por profesor, observador y estudiantes en la sistematización de la observación de las sesiones del curso (véase la tabla 3) en lo que se refiere al reconocimiento de la diversidad de poblaciones y de las exigencia que plantea esa diversidad para el aprendizaje de la matemática.

La interacción en línea no solo permitió el acceso y uso de los OvA, sino también amplió las formas de comunicación para dar apertura a discusiones sobre accesibilidad, reconocimiento de diversidad de poblaciones y desarrollo de posiciones que posiblemente consoliden la identidad de profesor de matemáticas, la herramienta del Atutor en la que se manifestaron estas formas de comunicación fue el foro.

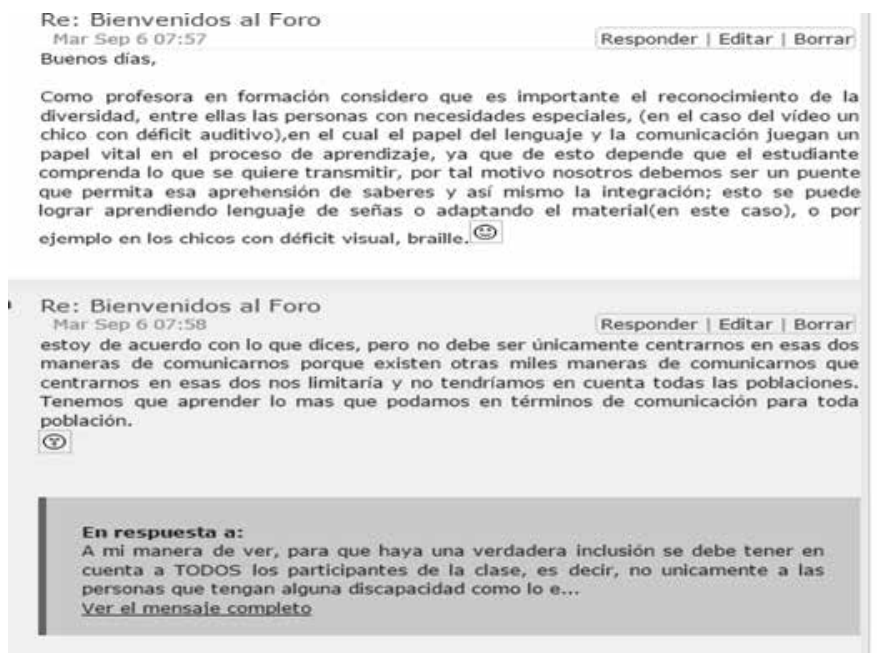

Imagen 10. Discusión de los estudiantes en el foro del curso Práctica II

La relación entre accesibilidad de los diseños y reconocimiento de la diversidad de poblaciones también se detectó en el laboratorio de la arquitectura en datos provenientes de las evaluaciones del curso por parte de los estudiantes. Los siguientes relatos son tomados de este análisis: 
Grupo 1:

El curso Práctica Intermedia II tuvo un énfasis en la implementación y accesibilidad de los recursos didácticos para la enseñanza de las matemáticas en poblaciones diversas... desde el principio se implementó una plataforma (ATutor) cuyo objetivo era concientizarnos acerca de la diversidad de las poblaciones, con las cuales en nuestro ejercicio como docentes podremos encontrarnos. Con esto, a medida que transcurría la práctica los miembros del curso iban desarrollando actividades, documentos y materiales teniendo en cuenta tanto el objeto matemático a enseñar, como las diversas poblaciones a las cuales pueden estar dirigidas nuestras producciones. (Bisbicus y Luna, 2016, p. 275).

\section{Grupo 2:}

Es importante decir que el estudiante como profesor no debe pensar solo en el uso de recursos didácticos, sino también, en la elaboración de estos y al Ilevar acabo esta tarea, debe pensar en la accesibilidad y la usabilidad del recurso; hablar de accesibilidad refiere a la facilidad de uso del recurso, sopesando la eficacia y la eficiencia para el uso de cualquier persona de acuerdo a sus capacidades, [...] (Culman y Quintero, 2016, p. 9).

La dinámica desarrollada a partir de la arquitectura proyecta los resultados anteriores al ámbito de la reflexión metadidáctica y del refinamiento de la práctica como experiencia de constelación. En este ámbito se espera consolidar la generación de conjeturas didácticas y teorías para mejorar posteriores diseños.

\section{Conclusiones}

En lo desarrollado hasta el momento en el proyecto de investigación "Desarrollo didáctico y tecnológico en escenarios didácticos para la formación de profesores que acogen la diversidad" se puede evidenciar la importancia y las posibilidades de análisis y validación de las hipótesis didácticas que fundamentan el diseño de ambientes y ova, a partir de la estructura de la arquitectura de validación.

Los tres ámbitos para el refinamiento de la práctica didáctica se caracterizan por ser escenarios para el desempeño de roles apropiados para las prácticas y la emergencia de los procesos que le otorgan idoneidad a estas. A su vez facilitan la constitución de la identidad profesional desde la realización, la gestión y la reflexión metadidáctica. Los resultados de investigación se constituyen en evidencia de la adecuada articulación de los tres enfoques presentes: la investigación en diseño, los experimentos de enseñanza y las comunidades de práctica. 
En el ámbito de realización, la investigación en diseño y el experimento de enseñanza permitieron el diseño del curso fuente y de los tres ambientes de aprendizaje. Se evidencia que el formador de profesores fue autor en la creación del ambiente de su curso, es decir, no fue un ejecutor de un diseño previamente elaborado.

En el ámbito de gestión de la investigación, las comunidades de práctica y la investigación en diseño brindaron la posibilidad de sistematizar, comprender y analizar los diseños y la implementación de los ambientes de aprendizaje, a partir de las valoraciones de profesores, estudiantes y observadores en el proceso de implementación desde los instrumentos de observación y evaluación, y desde las discusiones y el análisis dentro de la comunidad de investigadores, donde es posible la evolución de los diseños didácticos y el aprendizaje de todos con todos.

La interacción entre los ámbitos de realización y gestión fue desarrollada por la movilización de información permitida por los corredores y los actores que circularon en ellos, en este caso investigadores y profesores.

La arquitectura es una estructura de naturaleza didáctica para la evaluación de diseños didácticos; es producto de esta investigación exploratoria, por consiguiente se requerirá continuar las investigaciones sobre estructuras de este tipo con el fin de fortalecer la formación de profesores de matemáticas para el acogimiento de la diversidad de poblaciones.

\section{Referencias}

Association of Mathematics Teacher Educators. (2017). Standards for preparing teachers of mathematics. Disponible en amte.net/standards.

Biggs J. y Tang C. (2007). Teaching for quality learning at university. Berkshire: SRHE \& Open University Press.

Bisbicus, L. y Luna, J. (2016). Recorriendo los conceptos de área y perímetro a través de materiales didácticos en cuarto grado. Bogotá: Universidad Distrital Francisco José de Caldas.

Blondet, L. y Nascimento, R. (2004). Learning theory and instruction design using learning objects. Journal of Educational Multimedia and Hypermedia, 343-370. ISSN 1055-8896.

Brown, A. (1992). Design experiments: Theoretical and methodological challenges in creating complex interventions in classroom settings. The Journal of the Learning Sciences, 2(2), 141-178.

Bynner, J. y Parsons, S. (2005). Does numeracy matter more? Londres: Institute of Education, University of London.

Calderón, D. y León, O. (2015). Elementos para una didáctica del lenguaje y las matemáticas en estudiantes sordos de niveles iniciales. Bogotá: Fondo de publicaciones Universidad Distrital Francisco José de Caldas. 
Cast (2011). Universal Design for Learning Guidelines version 2.0. Wakefield, MA: autor.

Cisternas, T. (2011). La investigación sobre formación docente en Chile. Territorios explorados e inexplorados. Calidad en la educación, 23, 131-164.

Clements, D. y Sarama, J. (2009). Early childhood mathematics education research. Nueva York: Routledge.

Cobb, P. \& Gravemeijer, K. (2008). Experimenting to support and understand learning processes. En A. E. Kelly, R. A. Lesh, y J. Y. Baek (eds.) Handbook of design research methods in education: Innovations in science, technology, engineering, and mathematics learning and teaching, (pp. 68-95). Mahwah, NJ: Lawrence Erlbaum Associate.

Culman M. y Quintero, M. (2016). Midiendo figuras tridimensionales. Bogotá: Universidad Distrital Francisco José de Caldas.

Díaz-Barriga, F. (2006). Principios de diseño instruccional de entornos de aprendizaje apoyados en TIC: un marco de referencia sociocultural y situado. Tecnología y Comunicación Educativa, 41. Recuperado de http://investigacion.ilce.edu.mx/tyce/41/art1.pdf.

Dresh, A.; Pacheco, D. \& Valle, J. (2015). Design science research. A method for science and tecnology. Nueva York: Springer.

Fals B., O. (1978). Por la praxis: el problema de cómo investigar la realidad para transformarla. En H. Moser y H. Ornaner (eds). Internationale Aspekte der Aktionsforschung. Munich: Kösel.

Fernández, C.; Llinares, S. y Valls, J. (2011). Características del desarrollo de una mirada profesional en estudiantes para profesor de matemáticas en un contexto B-learning. Acta Scientiae, 13(1), 9-29.

Flores, P. (1998). Concepciones y creencias de los futuros profesores sobre las matemáticas, su enseñanza y aprendizaje. Investigación durante las prácticas de enseñanza. Granada: Comares.

Frenkel, E. (2015). Amor y matemáticas. Madrid: Ariel.

García-García, F. (2006). Contenidos educativos digitales: construyendo la sociedad del conocimiento. Red Digital. Revista de Tecnologías de la información y comunicación educativa, 6.

García-Oliveros, G. y Monteho-Rozo, J. (2009). Las relaciones entre evaluación y el orden social en la clase de matemáticas. Un estudio en una clase de álgebra. Voces y Silencios. Revista Latinoamericana de Educación, 128-138.

Gil-Chaves, D. (2016). Una mirada sistémica de los programas de formación de profesores de matemáticas. Revista Horizontes Pedagógicos, 18(1), 110-125.

Göbel H. y Cronholm S. (2012). Design science research in action-experiences from a process perspective. A Pre-ecIs and AIS sIG Prag Workshop on Iт Artefact Design y Workpractice Intervention. Barcelona. 
Godino, J. D. (2009). Categorías de análisis de los conocimientos del profesor de matemáticas. Revista Iberoamericana de Educación Matemática. 20, 13-31.

Godino, J. D. (2014). Indicadores de idoneidad didáctica de procesos de enseñanza y aprendizaje de las matemáticas. Conferencia presentada en Ciclo de conferencias en Educación Matemática de Gemad (8 de noviembre de 2014). Bogotá.

Gómez, P. y Lupiáñez, J. (2007). Trayectorias hipotéticas de aprendizaje en la formación inicial de profesores de matemáticas de secundaria. PNA, 1(2), 79-98.

Lafrancesco G. (1996). Nueve problemas de cara a la renovación educativa. Alternativas de solución. Santafé de Bogotá: Libros y Libros.

Laurillard, D. (2012). Teaching as a design science. Building pedagogical patterns for learning and technology. Nueva York: Routledge.

León, O. (2016). Aplicación del modelo mva-N en la comunidad cam. En Á. López y Mota, y O. León (Eds.), Modelo de validación ALTER-NATIVA de objetos virtuales de aprendizaje en escenarios naturales (pp. 164210). Bogotá: Universidad Distrital Francisco José de Caldas.

León, O.; Calderón, D.; García, A. y Martínez, M. (2016). Didactics and teaching with accessibility and affectivity in higher education. Proceedings of the 7th International Conference on Software Development and Technologies for Enhancing Accessibility and Fighting Info-exclusion (pp. 99-104). Doi: 10.1145/3019943.3019958.

León, O.; Díaz, F. y Guilombo, M. (2014). Diseños didácticos y trayectorias de aprendizaje de la geometría de estudiantes sordos, en los primeros grados de escolaridad. Revista Latinoamericana de Etnomatemática, 7(2), 4-23.

León, O.; Medina, R.; Saiz, M.; Bonilla, M.; Romero, J.; Gil, D. et al. (2013). Relaciones entre "diseño para todos" y "diseño con todos" en formación de profesores de matemáticas. Para uma Formacao Virtual Acessivel e de Qualidade (pp. 162-169).

León, O. y López y Mota, Á. (2016). Modelo de validación ALTER-NATIVA de objetos virtuales de aprendizaje en escenarios naturales. Bogotá: Universidad Distrital Francisco José de Caldas.

Ministerio de Educación Nacional (2016). Revisión de políticas nacionales de educación: educación en Colombia. Bogotá: autor. Recuperado de www.mineducacion.gov.co/1759/articles-356787_recurso_1.pdf.

Molina, M. y Castro, E. (2006). Desarrollo del pensamiento relacional trabajando con igualdades numéricas. Indivisa, Boletín de Estudios e Investigación, monografía Iv, 91-103.

Molina, M.; Castro, E.; Molina, L. \& Castro, E. (2011). Un acercamiento a la investigación de diseño a través de los experimentos de enseñanza. Enseñanza de las Ciencias, 29(1), 75-88. 
Munro, M. \& Kenny, C. (2008). Standards for learning objects and learning designs. En Lockyer, L. et al. (eds.). Handbook of research on learning design and learning objects: Issues, applications and technologies (vol. 2, pp. 827-850). Hershey (Pennsylvania): Information Science Reference.

Ponte, J. y Chapman, O. (2006). Mathematics teachers' knowledge and practices. En A. Gutiérrez y P. Boero (eds.). Handbook of Research on the Psychology of Mathematics Education: Past, Present and Future (pp. 461-494).

Porlan, R. (1993). Constructivismo y escuela. Hacia un modelo de enseñanza-aprendizaje basado en la investigación. Sevilla: Díada.

Prodromou L. (1995). The backwash effect: From testing to teaching. ELT Journal, 49(1), 13-25.

Reyes, M. R.; Brackett, M. A.; Rivers, S. E.; White, M. y Salovey, P. (2012). Classroom emotional climate, student engagement, and academic achievement. Journal of Educational Psychology, 104(3), 700-712.

Simon, M. y Tzur, R. (2004). Explicating the role of mathematical tasks in conceptual learning: An elaboration of the hypothetical learning trajectory. Mathematical Thinking and Learning, 6(2), 91-104.

Sinclaire, J. K. (2014). An empirical investigation of student satisfaction with college courses. Research in Higher Education Journal, 22, 1-21.

Wenger, E. (2001). Comunidades de práctica. Aprendizaje, significado e identidad. Barcelona: Paidós. 